\title{
Development of Nano-tetragonal Zirconia-Incorporated Ni-P Coatings for High Corrosion Resistance
}

\author{
S. M. A. Shibli ${ }^{1}$ K. S. Chinchu ${ }^{1} \cdot$ M. Ameen Sha ${ }^{1}$ \\ Received: 18 May 2018/Revised: 1 August 2018/Published online: 29 September 2018 \\ (C) The Chinese Society for Metals and Springer-Verlag GmbH Germany, part of Springer Nature 2018
}

\begin{abstract}
Pure tetragonal 10-20-nm-size zirconia-based Ni-P composite coating was developed. The physicochemical and electrochemical characteristics including corrosion resistance of the coating were investigated. The Ni-P-nano-tetragonal zirconia coating was partially crystalline having face-centered cubic phase. The coating had very high corrosion resistance due to its dense compact morphology and low surface roughness. The Ni-P-nano-tetragonal zirconia coatings exhibited a cathodic shift of open-circuit potential (OCP) in the range from -0.340 to $-0.520 \mathrm{~V}$. A high polarization resistance of the order of $13.2 \mathrm{k} \Omega / \mathrm{cm}^{2}$ and low corrosion current density of $3.9 \mu \mathrm{A} / \mathrm{cm}^{2}$ were achieved due to the effective incorporation of zirconia into the coating.
\end{abstract}

Keywords Composites $\cdot$ Corrosion resistance $\cdot$ Substrates $\cdot \mathrm{Ni}-\mathrm{P} \cdot \mathrm{ZrO}_{2}$

\section{Introduction}

Electroless Ni-P coatings are extensively used for surface engineering and industrial applications due to their unique properties such as high wear and abrasion resistance, hardness, corrosion resistance. Mild steel tubular articles used for oil and gas production are protected by applying a layer of electroless Ni-P coating on its surface $[1,2]$. The incorporation of second-phase particles or composites in Ni-P matrix can further improve the mechanical and physical properties of the coatings [3]. Depending on the end application, the criteria of selection of the composite may be fixed [4]. The incorporation of functional particles into the Ni-P matrix results in electroless $\mathrm{Ni}-\mathrm{P}$ composite coatings with dual characteristics of both the base Ni-P matrix and the functional additive [4]. The solid particles

Available online at http://link.springer.com/journal/40195

Electronic supplementary material The online version of this article (https://doi.org/10.1007/s40195-018-0823-4) contains supplementary material, which is available to authorized users

S. M. A. Shibli

smashibli@yahoo.com

1 Department of Chemistry, University of Kerala, Kariavattom Campus, Thiruvananthapuram, Kerala 695 581, India such as oxides, carbides and other ceramics have been extensively studied in recent years, as second-phase additives for Ni-P matrix. The corrosion of the coatings in the presence of halide ions can be effectively hindered by incorporating ceramic oxide coatings with excellent chemical stability [5]. Further reduction in particle size of the composite into the nano-size can lead to development of new-generation nano-composite coatings. Synthesis and specific use of reinforcing particles in nano-metric range is a technological and industrial interest for the development of new composite materials with unique, well-controlled and improved properties. The mechanical and tribological properties of the coatings can be improved by the uniform dispersion of such composites [6]. The corrosion resistance of the electroless nano-composite coatings such as $\mathrm{Ni}-\mathrm{Fe}-$ $\mathrm{P}$ [7], Ni-P-TiO 2 [8], ternary alloys of $\mathrm{Cu} / \mathrm{Zn} / \mathrm{Sn}$ with $\mathrm{Ni}-\mathrm{P}$ coating [9], Ni-P-nano-SiO ${ }_{2}$ composite coating [10], NiP-PTFE [11], SiC-NiP coatings [12] and Ni-Mo-P [13] would be better than that of the bare $\mathrm{Ni}-\mathrm{P}$ coating. The nano-composite incorporation can also enhance tribological and metallurgical properties of the $\mathrm{Ni}-\mathrm{P}$ coatings.

Zirconia is a well-known ceramic material having applications in various fields of industry [14, 15]. Nanotechnology of zirconia-based ceramic material is of great interest in various sectors of engineering and technology. Theoretical studies of the formation of $\mathrm{ZrO}_{2}$ materials [16], microstructure and corrosion resistance of the material 
[17], variety of synthetic strategies [18, 19] are mainly focused by many researchers. Under thermal equilibrium conditions, zirconia exists in three well-known structural forms, namely monoclinic (is stable up to $1170{ }^{\circ} \mathrm{C}$ ), tetragonal (exists between 1170 and $2370{ }^{\circ} \mathrm{C}$ ) and cubic (exists above $2370{ }^{\circ} \mathrm{C}$ ) [20-22]. High-pressure orthorhombic form of zirconia is metastable at atmospheric pressure and reverts to the monoclinic form by mechanical grinding [23, 24]. Reduction of the particle size of zirconia to nanometer regime stabilizes its high-temperature phases [16]. Tetragonal zirconia has gained much interest due to its excellent thermal stability, chemical resistance, fracture toughness, high hardness, high strength, etc. [25-28]. Moreover, the grain size also plays a significant role in the thermal stability of zirconia polymorphs. The tetragonal phase of zirconia becomes energetically favorable as the grain size decreases below a critical value due to energy crossover [29]. It is also proved that the tetragonal phase is the most resistant phase of zirconia [30].

Nano-size effect has a key role in controlling the phase stability of the nano-composite under ambient conditions [31]. The formation of metastable crystalline structures is only possible for the bulk materials under conditions of high temperature and pressure, whereas the decrease in crystallite size of these materials leads to the formation of metastable crystal structures in the nano-size regime [32]. The large internal pressure in the nano-crystallites (due to its high surface curvature) and hardening of the soft phonon modes (due to the disorder in the surface atomic arrangements and the crystal symmetry) stabilize the metastable phases [33-36]. The tetragonal zirconia exhibits high hardness comparable than steel and possesses high chemical stability [24, 37, 38]. In addition, zirconia in all its physical and structural forms is a biocompatible material and does not cause any cytotoxicity [39]. Yang et al. [40] synthesized $\mathrm{Ni}-\mathrm{P}-\mathrm{ZrO}_{2}$ nano-composite coating and studied its mechanical properties and formation mechanism. Szczygieł et al. [41] reported surface morphology and structure of an electroless $\mathrm{Ni}-\mathrm{W}-\mathrm{P}-\mathrm{ZrO}_{2}$ coating by using a glycine bath. Zielinska et al. [42] studied the effect of the addition of various surfactants during deposition of a $\mathrm{Ni}-\mathrm{P}-$ nano- $\mathrm{ZrO}_{2}$ composite coating and also its effect on the morphology and composition of each layer of the coating. Song et al. [43] developed $\mathrm{Ni}-\mathrm{P}-\mathrm{ZrO}_{2}$ (nanometer) composite coating with high wear and corrosion resistance. However, the corrosion resistance characteristics of Ni-P-nano-tetragonal zirconia coatings on the steel have not yet been studied.

The present study aimed to explore nano-tetragonal zirconia prepared by wet thermal decomposition for enhancing the corrosion resistance of $\mathrm{Ni}-\mathrm{P}$ coatings in $\mathrm{NaCl}$ medium. The characteristics of the prepared nanotetragonal zirconia, the effect of incorporation of such nano-tetragonal zirconia into the $\mathrm{Ni}-\mathrm{P}$ coating, and the enhancement in physicochemical and electrochemical characteristics including the corrosion resistance of the coating were studied. The corrosion prevention characteristics of the interior layers of the coating were also studied.

\section{Experimental}

\subsection{Synthesis and Characterization of Nano- tetragonal Zirconia}

Zirconia $\left(\mathrm{ZrO}_{2}\right)$ nanoparticles were prepared by a wet thermal decomposition method. The required amount of zirconium oxychloride ( $2 \mathrm{~g}$ ) was mixed in isopropanol $(5 \mathrm{~mL})$. After thorough mixing, the slurry was evaporated to dryness followed by heating at $120{ }^{\circ} \mathrm{C}$ for $1 \mathrm{~h}$. Thermal decomposition of the precursor was ensured by heating the zirconia powder in a muffle furnace at $450{ }^{\circ} \mathrm{C}$ for $1 \mathrm{~h}$ with a heating rate of $1{ }^{\circ} \mathrm{C} / \mathrm{min}$. The phase characteristics of the prepared nano-tetragonal zirconia were analyzed using a Philips Xpert MPD X-ray powder diffractometer using $\mathrm{Cu} K_{\alpha}$ radiation $(\lambda=1.540 \AA)$. UV-visible reflectance analysis was carried out by using Shimadzu UV-2450 analyzer over a wavelength range from 200 to $900 \mathrm{~nm}$. The phase structure of the prepared nano-tetragonal zirconia was confirmed from the results of selected area electron diffraction (SAED) and high-resolution transmission electron microscopy (HRTEM, TEM-JEOL JEM 2100) analysis. The particle size of the prepared nano-tetragonal zirconia was also analyzed accordingly. The bonding characteristics and functional groups in the particle were analyzed by using IR absorption spectrum (PerkinElmer Spectrum2) recorded in the range of $400-4000 \mathrm{~cm}^{-1}$ with $\mathrm{KBr}$ pellets. The thermal stability of the prepared nanotetragonal zirconia was analyzed from the results of X-ray diffraction patterns of the nano-tetragonal zirconia annealed at $250{ }^{\circ} \mathrm{C}$ for $1 \mathrm{~h}$ and by thermogravimetricdifferential thermal gravimetric analysis (TG-DTG, PerkinElmer, Diamond TG/DTA, PerkinElmer STA 6000). The stability of the nano-tetragonal zirconia in the $\mathrm{Ni}-\mathrm{P}$ bath was analyzed by X-ray diffraction (XRD) spectrum of the residue left in the bath after the deposition process. It was further confirmed by Fourier transform infrared (FTIR, FT-MIR Bruker Tensor 27 spectrophotometer) and UVvisible spectroscopy. Morphological and compositional characteristics of the prepared nano-tetragonal zirconia were analyzed by field emission scanning electron microscopy (FESEM, NOVA Nano SEM 450) equipped with an EDS instrument. 


\subsection{Evaluation of Ni-P-Nano-tetragonal Zirconia Coatings}

$\mathrm{Ni}-\mathrm{P}-$ nano-tetragonal zirconia coatings were prepared on pretreated mild steel substrates by adopting a reported procedure [44]. The bath contains $100 \mathrm{~mL}$ nickel sulfate solution $(30 \mathrm{~g} / \mathrm{L})$ and $2.5 \mathrm{~g}$ succinic acid, and its $\mathrm{pH}$ was adjusted to 4.5 by adding ammonia. The bath temperature was maintained at $85{ }^{\circ} \mathrm{C}$ throughout the deposition process. An amount of $2.5 \mathrm{~g}$ sodium hypophosphite was added into the bath just before inserting the pretreated substrate into the bath. The substrate was pretreated in series first by acid etching with $3 \%(\mathrm{v} / \mathrm{v}) \mathrm{HCl}$ followed by $5 \%(\mathrm{w} / \mathrm{v}) \mathrm{NaOH}$. Then, the substrate was activated using $10 \mathrm{~g} / \mathrm{L} \mathrm{SnCl}_{2}$ in $40 \mathrm{~mL} / \mathrm{L} \mathrm{HCl}$ and then sensitized using $1 \mathrm{~g} / \mathrm{L} \mathrm{PdCl}_{2}$ in $10 \mathrm{~mL} / \mathrm{L} \mathrm{HCl}$ solution. Varying amount of nano-tetragonal zirconia, viz. $1 \mathrm{~g} / \mathrm{L}, 2 \mathrm{~g} / \mathrm{L}, 5 \mathrm{~g} / \mathrm{L}$ and $10 \mathrm{~g} / \mathrm{L}$, was added into the Ni-P bath and stirred constantly $(250 \mathrm{rpm})$ during the coating process for developing Ni-P-nano-tetragonal zirconia coatings.

The variations in the deposition potential of the mild steel substrates during the course of the deposition were monitored as a function of time using saturated calomel electrode (SCE) as the reference electrode. The deposition rate of the coatings was measured from the weight difference of the mild steel substrates before and after the deposition process. Micro-hardness of the coatings was analyzed as per ASTM-E-384-99, and thickness was calculated from the method as described elsewhere [44]. Ferroxyl reagent test was conducted for accessing the porosity of the developed coatings. Morphological and compositional characteristics of the coatings were examined using a scanning electron microscope (SEM, JEOL JSM-840A) and an energy-dispersive X-ray spectroscope (EDS). Topographical characteristics of the coatings were examined by Bruker-Dimension edge with a scan assist AFM (atomic force microscope). Phase characteristics of the coatings were examined using an X-ray diffractometer with $\mathrm{Cu} K_{\alpha}$ radiation. The corrosion resistance characteristics and stability of the coatings in a corrosive medium were analyzed by open-circuit potential (OCP) analysis and potentiodynamic polarization method with a three-electrode system. Three-electrode setup consists of a unit area of the coating as working electrode, SCE as the reference electrode and platinum mesh $\left(3 \mathrm{~cm}^{2}\right)$ as the counter electrode. Tafel polarization and impedance analysis of the coatings were carried out using AUTOLAB PGSTAT 30 potentiostat in $3.5 \%(\mathrm{w} / \mathrm{v}) \mathrm{NaCl}$. The Tafel polarization was carried out between -1.000 and $0.250 \mathrm{~V}$ with a scan rate of $20 \mathrm{mV} / \mathrm{s}$. EIS analysis was performed with a $10-\mathrm{mV}$ perturbation amplitude AC signal over a frequency range from $1 \mathrm{MHz}$ to $10 \mathrm{mHz}$.
Corrosion current density $\left(I_{\text {corr }}\right)$ and corrosion rate $(\mathrm{CR})$ of the coatings were calculated using Stern-Geary equation and Faraday law, respectively

$I_{\text {corr }}=\left[\beta_{\mathrm{a}} \beta_{\mathrm{c}} / 2.303\left(\beta_{\mathrm{a}}+\beta_{\mathrm{c}}\right)\right]\left[1 / R_{\mathrm{p}}\right]$

where $\beta_{\mathrm{a}}$ and $\beta_{\mathrm{c}}$ are the anodic and cathodic Tafel slopes and $R_{\mathrm{p}}$ is the polarization resistance. $R_{\mathrm{p}}=(\Delta E / \Delta i)_{\Delta E \rightarrow 0}$, where $\Delta E$ is the polarization potential and $\Delta i$ is the polarization current.

$\mathrm{CR}=K\left(i_{\text {corr }} / \rho\right) \mathrm{EW} \quad[46,47]$

where $K$ is a constant and is taken as $3.27 \times 10^{-3} \mathrm{~mm} \mathrm{~g} /$ ( $\mu \mathrm{A} \mathrm{cm}$ year), $\rho$ is the density of the substrate, and EW is the equivalent weight of the substrate.

The stability of the coatings in aggressive conditions was examined using salt spray analysis. Anodic dissolution studies of the developed coatings using different current densities $\left(20 \mathrm{~mA} / \mathrm{cm}^{2}, 50 \mathrm{~mA} / \mathrm{cm}^{2}\right)$ were employed to analyze the corrosion characteristics of the internal layers of the coatings.

\section{Results and Discussion}

\subsection{Characterization of the Nano-tetragonal Zirconia}

Figure 1a shows the X-ray diffractograms of nano-tetragonal zirconia. Some broad peaks were observed in the XRD spectra, which indicated small size of the nano-crystallites in the nano-tetragonal zirconia [39]. The nanoscale crystalline growth in the nano-tetragonal zirconia was an intricate process controlled by precursor concentration and reaction temperature $[48,49]$. Strong and characteristic XRD peaks (JCPDS 42-1164) at 2 theta $(2 \theta)$ values of $30.59^{\circ}, 37.60^{\circ}, 50.69^{\circ}$ and $60.66^{\circ}$ were attributed to the (111), (200), (220) and (311) diffraction planes of tetragonal zirconia [30, 50]. It is difficult to distinguish the

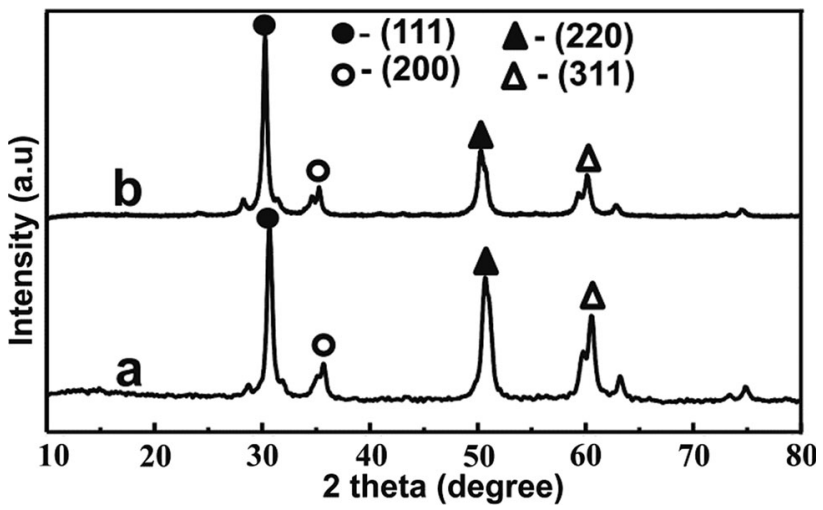

Fig. 1 XRD patterns of the (a) nano-tetragonal zirconia (b) nanotetragonal zirconia recovered from the electroless Ni-P bath 
tetragonal and cubic phases of zirconia as they have similar lattice parameters [51-53]. However, tetragonal structures of zirconia can be distinguished with respect to the characteristic splitting of Bragg profiles from ' $c$ ' index planes [24, 54-56]. Cubic phase exhibits only single diffraction peak [57]. The split Bragg peaks of nano-tetragonal zirconia overlapped with one another due to peak broadening. Hence, the XRD spectrum was subjected to thorough examination. Two doublets were observed in the spectra. They were: (1) at $2 \theta$ values $30.59^{\circ}$ and $30.80^{\circ}$ with relative intensities of $100.00 \%$ and $84.90 \%$, (2) at $2 \theta$ values $50.69^{\circ}$ and $51.09^{\circ}$ with relative intensities of $75.58 \%$ and $58.92 \%$, respectively. These observations revealed the formation of nano-tetragonal zirconia by the preparation process in accordance with the reports of Sherafat and Antunes [58]. The average crystallite size of the nano-tetragonal zirconia was calculated using Scherrer equation. It was found to be $190 \mathrm{~nm}$.

The phase structure of the prepared nano-tetragonal zirconia was confirmed by selected area electron diffraction (SAED) and HRTEM (Fig. 2A, B). The equidistant parallel fringes portrayed the single crystalline nature of the nano-tetragonal zirconia [25]. Lattice fringes with an inter-planar spacing of $0.31 \mathrm{~nm}$ were attributed to the lattice $d$-spacing of (101) plane of prepared nano-tetragonal zirconia [59]. The few clear spots observed in the SAED pattern indicated the presence of crystallites having sufficient size to diffract [39]. A circular SAED pattern observed for nano-tetragonal zirconia was in accordance with the reports [60, 61]. The HRTEM and electron diffraction investigations evidenced the formation of tetragonal zirconia phase. A well-defined spherical zirconia nanoparticle structure was clearly observed, and the particle size was found to be within the range of 10-20 nm. The particle size of the nano-tetragonal zirconia plays a significant role in its phase stabilization [25]. The monoclinic zirconia phase can be stabilized within the particle size range greater than or equal to $20 \mathrm{~nm}$, the tetragonal zirconia which was stable in the range of 7-20 nm, and cubic phase gets stabilized within the particle size range less than or equal to $6 \mathrm{~nm}$ [25]. In the present case, zirconia got stabilized as tetragonal phase.

The EDS spectrum of the nano-tetragonal zirconia is shown in Fig. 2C. The element analysis confirmed the presence of zirconium and oxygen in the prepared nanotetragonal zirconia [48]. No other peaks were observed, indicating the high purity and also the completion of thermal decomposition of zirconium oxychloride during the preparation of nano-tetragonal zirconia. Figure 2D shows the FESEM image of the nano-tetragonal zirconia. Scattered but dense aggregates of the nano-tetragonal zirconia with an irregular morphology were clearly observed. As the nano-tetragonal zirconia as dense aggregate forms, it needs a thorough dispersion of the particles in the electroless Ni-P bath during the deposition process. This was

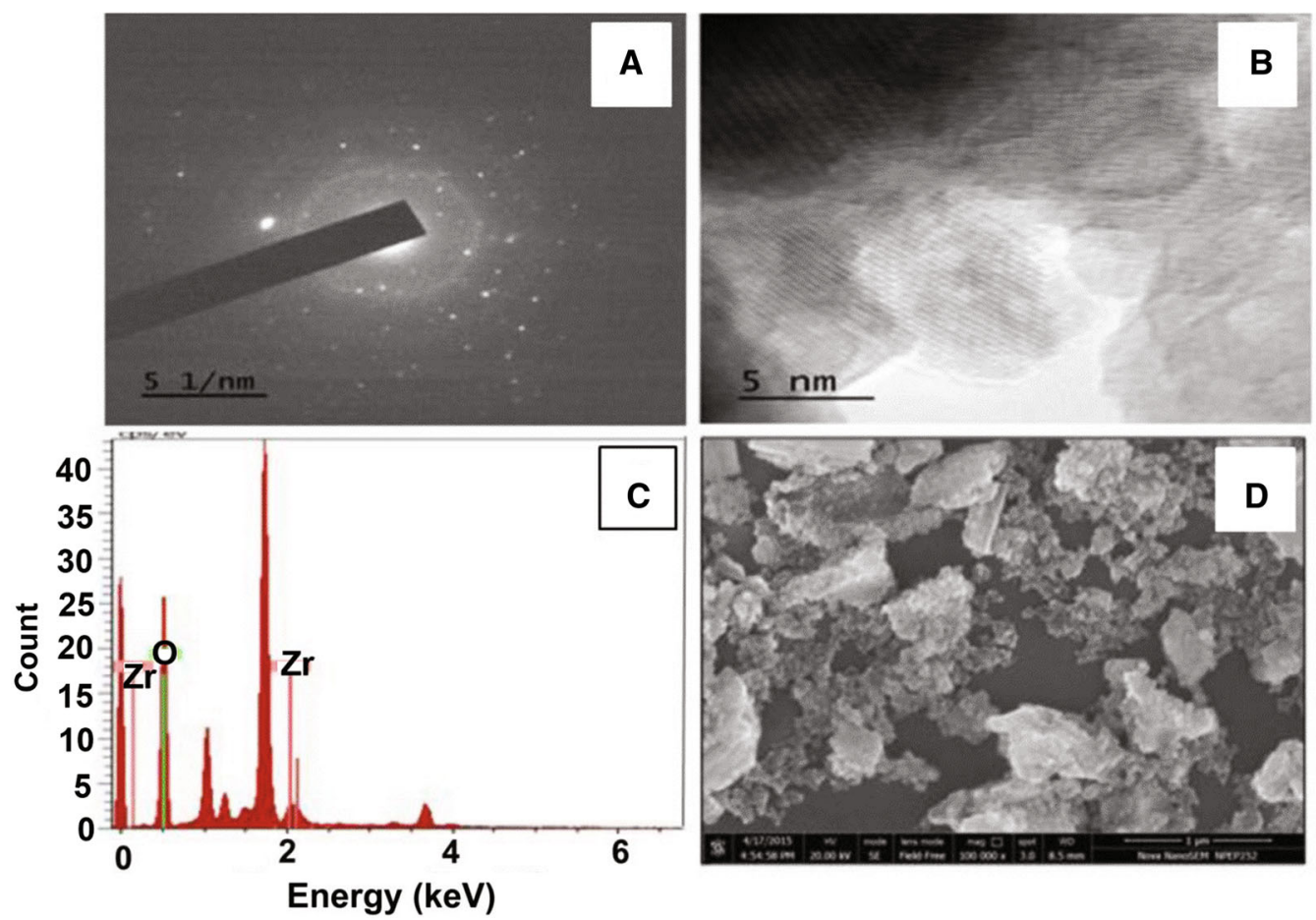

Fig. 2 A SAED pattern and B high-resolution TEM image of nano-tetragonal zirconia, $\mathbf{C}$ the EDS spectrum of the synthesized nano-tetragonal zirconia, D FESEM image of the nano-tetragonal zirconia (magnification 100,000 $\times$ ) 
achieved by adding nano-tetragonal zirconia within the $\mathrm{Ni}-$ $\mathrm{P}$ bath for $30 \mathrm{~min}$ prior to the deposition process.

The UV-visible spectrum of the nano-tetragonal zirconia is shown in Fig. 3III-a. The UV-visible spectrum indicated that the nano-tetragonal zirconia was active in the UV-visible region. A single absorption maximum at around $210 \mathrm{~nm}$ and absorption edge around $260 \mathrm{~nm}$ revealed the existence of nano-tetragonal zirconia [62]. FTIR spectrum of the nano-tetragonal zirconia is shown in Fig. 3IV-a. A broad band with a peak at $557 \mathrm{~cm}^{-1}$ and a shoulder peak at $645 \mathrm{~cm}^{-1}$ was observed that revealed the presence of $\mathrm{Zr}-\mathrm{O}$ vibrations in the nano-tetragonal zirconia [30, 63-67]. IR bands at $3428 \mathrm{~cm}^{-1}$ and $1627 \mathrm{~cm}^{-1}$ in the spectrum were attributed to the stretching and bending vibrations of the $\mathrm{O}-\mathrm{H}$ bond due to the presence of physisorbed water molecules present on the surface of the nanotetragonal zirconia [63-65], whereas the weak band at $1384 \mathrm{~cm}^{-1}$ was attributed to the absorption of non-bridging $\mathrm{O}-\mathrm{H}$ groups [50]. Sharp band around $746 \mathrm{~cm}^{-1}$ was absent, which ruled out the presence of monoclinic zirconia in the nano-zirconia [25]. Thus, the FTIR spectra further confirmed the formation of nano-tetragonal zirconia with some physisorbed water molecules.

\subsection{Stability of the Nano-tetragonal Zirconia}

Stability of the nano-tetragonal zirconia at high temperature condition was analyzed by comparing its XRD patterns before and after the annealing process. The XRD patterns are shown in Fig. 3I. All the XRD peaks of the nano-tetragonal zirconia, before (Fig. 3I-a) and after (Fig. 3I-b) annealing were found to be identical. This observation revealed the phase stability of the nano-tetragonal zirconia. The peak intensity of annealed nanotetragonal zirconia was slightly higher than that of nonannealed nano-tetragonal zirconia. This was due to the crystallite aggregation of the nano-tetragonal zirconia that occurred after the annealing process, which in turn resulted in grain coarsening leading to the formation of bigger crystallites [68].

Thermal stability of the prepared nano-tetragonal zirconia was further confirmed by thermogravimetric analysis. The TG-DTG curves of the nano-tetragonal zirconia are shown in Fig. 3II. Initial weight of the sample taken for the analysis was about $4.981 \mathrm{mg}$. The sample was heated at a rate of $25^{\circ} \mathrm{C} / \mathrm{min}$ within the temperature range of $50-900{ }^{\circ} \mathrm{C}$. The analysis was performed in an inert
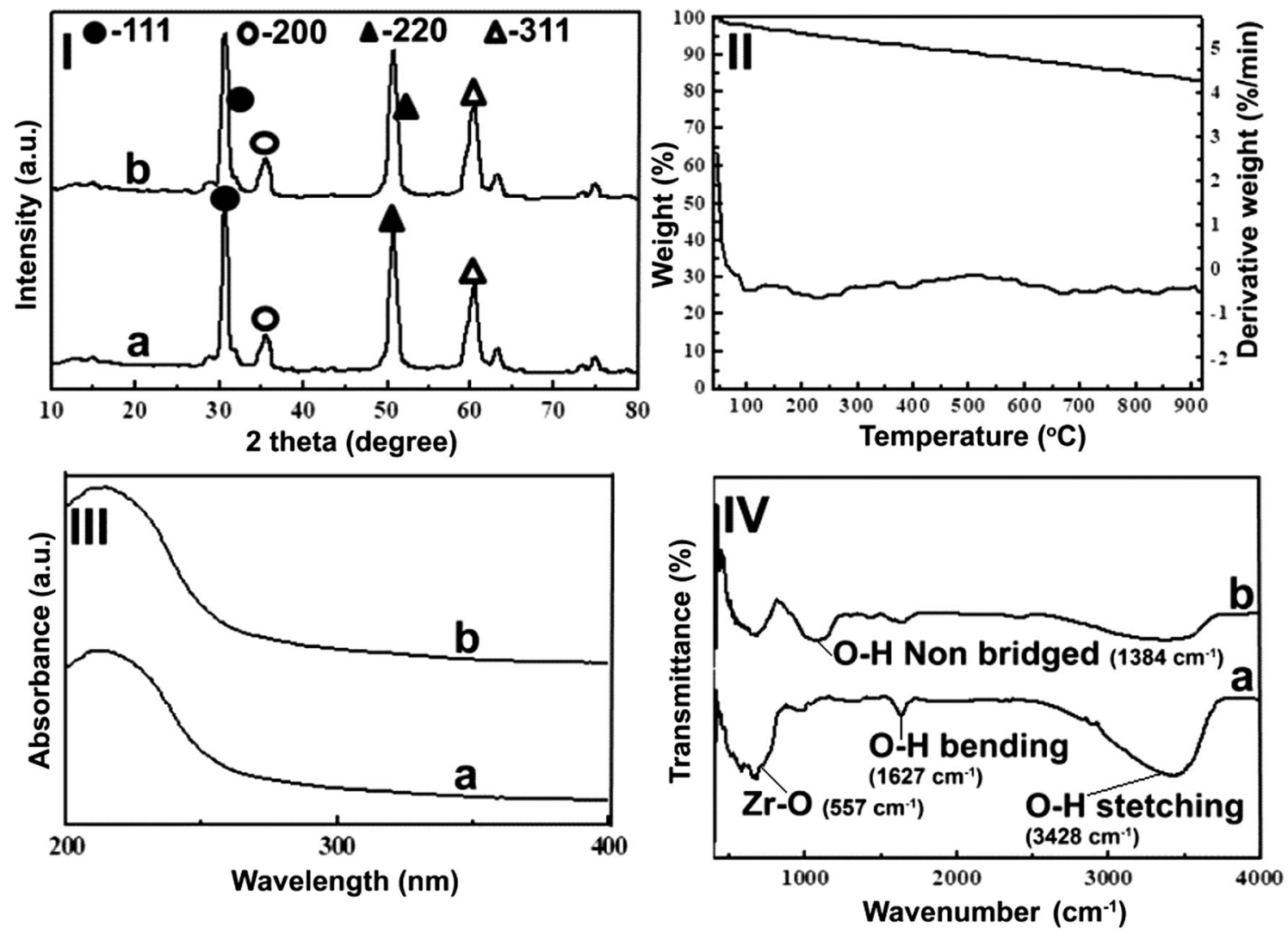

Fig. 3 I XRD patterns of (a) nano-tetragonal zirconia as prepared and (b) nano-tetragonal zirconia after annealing at a temperature of $250{ }^{\circ} \mathrm{C}$ for $1 \mathrm{~h}$, II TG-DTG curves of the nano-tetragonal zirconia, III UV-visible absorbance spectrum of (a) nano-tetragonal zirconia (b) nano-tetragonal zirconia from the electroless Ni-P bath, and IV FTIR spectra of (a) nano-tetragonal zirconia and (b) nano-tetragonal zirconia from the electroless $\mathrm{Ni}-\mathrm{P}$ bath 
atmosphere of $\mathrm{N}_{2}$. The total weight loss experienced by the nano-tetragonal zirconia in the entire temperature range was only less than $15 \%$ of the initial weight, which was due to either the removal of the solvent/water or due to the combustion of residual organics [69]. An endothermic peak in the DTG curve within $100{ }^{\circ} \mathrm{C}$ confirmed the evaporation of adsorbed water molecules. There was no considerable decrease in weight loss of the nano-tetragonal zirconia except that corresponding to the loss of physically adsorbed water molecules. No considerable variation in the TG curve with the weight loss revealed the thermal stability of the nano-tetragonal zirconia.

XRD pattern of the dried residue of the nano-tetragonal zirconia collected from Ni-P bath is compared with that of the as-prepared nano-tetragonal zirconia in Fig. 1a, b. Characteristic XRD patterns of the dried residue collected from the used electroless $\mathrm{Ni}-\mathrm{P}$ bath was similar to that of the as-prepared nano-tetragonal zirconia, revealing the stability of the nano-tetragonal zirconia in the electroless $\mathrm{Ni}-\mathrm{P}$ bath conditions. No other XRD peaks were observed in the spectra. It could be attributed to the absence of new phases. The number and intensity of the XRD peaks that remained same in both the cases also revealed the physical and chemical stability of the nano-tetragonal zirconia in the electroless deposition conditions. However, the residue retrieved after the deposition process from the bath exhibited a slight peak broadening due to the slightly amorphous nature of the residue collected from the electroless bath.

UV-visible absorbance spectrum of the dried residue is compared with that of the as-prepared nano-tetragonal zirconia (Fig. S1 in Supporting Information). The UVvisible absorbance spectrum of the residue (Fig. S1-b) exhibited a single absorption maximum around $210 \mathrm{~nm}$ with an absorption edge around $260 \mathrm{~nm}$ [62]. Both the absorption peak and edge exhibited by the residue were exactly like that of the as-prepared nano-tetragonal zirconia. No new absorption maximum in the UV-visible absorption spectra was observed, which indicated the absence of any phase transitions.

The FTIR spectrum of the dried residue is compared with that of the as-prepared nano-tetragonal zirconia (Fig. S2). The vibrational bands at $557 \mathrm{~cm}^{-1}$ and its shoulder band at $645 \mathrm{~cm}^{-1}$ that correspond to the $\mathrm{Zr}-\mathrm{O}$ vibrations of the dried residue of nano-tetragonal zirconia were merged and broadened in the FTIR spectrum from Ni-P bath [30, 66, 67]. The FTIR spectrum of the dried residue retrieved from the bath exhibited an additional broad band also around $1100 \mathrm{~cm}^{-1}$, ascribed to the presence of sulfate group in the filtered residue. It was caused by the usage of nickel sulfate solution as the source of $\mathrm{Ni}$ metal ions. The broadening of the peaks in the FTIR spectra of the filtered residue could be attributed to the deposition of a thin layer of electroless $\mathrm{Ni}-\mathrm{P}$ coating on the surface of the nanoparticles during the electroless deposition process. Thus, the physical and chemical stability of the nano-tetragonal zirconia, both in the electroless bath conditions and high temperature, was ensured prior to its incorporation into the Ni-P matrix.

\subsection{Characterization of the Ni-P-Nano- tetragonal Zirconia Coating}

\subsubsection{Evaluation of the Progress of Electroless Deposition}

The initial activation of electroless deposition could be easily accessed by monitoring the trend of variation in potential of electroless deposition and the rate of deposition. The trend of variation in potential due to the incorporation of different amounts of nano-tetragonal zirconia into the electroless bath is shown in Fig. S3. The potential of the mild steel substrate immersed either in the bare Ni-P or in the composite baths showed a shift in potential toward cathodic region as the deposition process was in progress. It might be due to the initial formation of a noble electroless coating on the surface of substrate. The coatings incorporated with nano-tetragonal zirconia exhibited a higher potential shift toward nobler region, compared to bare Ni-P coating. The shift in potential toward a noble region revealed the efficient incorporation of the nano-tetragonal zirconia into the Ni-P matrix [44]. It revealed that the Ni-P-nano-tetragonal zirconia coating was generated with a nobler surface than the bare Ni-P coating. The coating corresponding to the addition of $5 \mathrm{~g} / \mathrm{L}$ nano-tetragonal zirconia into the bath exhibited a higher cathodic potential shift during the electroless deposition revealing the highest deposition on the coating. The electroless coating was found to be uniform as the deposition potential was almost constant till the completion of the coating process.

The deposition rate of the coating was calculated from the weight difference of the substrate before and after the deposition process. The variation in the deposition rate of the electroless $\mathrm{Ni}-\mathrm{P}$ coatings after adding nano-tetragonal zirconia into the electroless bath is given in Fig. S4. The bare Ni-P coating exhibited a lower deposition rate of $22 \mathrm{mg} /\left(\mathrm{cm}^{2} / \mathrm{h}\right)$, and the rate increased $\left(36 \mathrm{mg} / \mathrm{cm}^{2} / \mathrm{h}\right)$ with the increase in concentration of the nano-tetragonal zirconia in the electroless bath up to $5 \mathrm{~g} / \mathrm{L}$. Above that concentration limit, the deposition rate was decreased. This evidenced a significant enhancement in the electroless deposition process by an optimum concentration $(5 \mathrm{~g} / \mathrm{L})$ of nano-tetragonal zirconia in the bath. All other coating parameters were retained constant for all coatings in order to avoid the effect of the parameters, the amount of 
complexing agent, time taken for coating, temperature and $\mathrm{pH}$ of the bath [70].

\subsubsection{Physicochemical Characterization of the Ni-P-Nano- tetragonal Zirconia Coating}

The bare Ni-P coating exhibited a lower hardness value in the range of 458-463 VHN and the thickness in the range of $10-11 \mu \mathrm{m}$, whereas $5 \mathrm{~g} / \mathrm{L} \mathrm{Ni-P-nano-tetragonal} \mathrm{zirco-}$ nia coating exhibited a higher hardness value in the range of 544-547 VHN and thickness in the range of 14-15 $\mu \mathrm{m}$ (Table 1). The co-deposited nano-tetragonal zirconia particles acted as new nucleation sites for nickel clusters and hence stopped their growth, resulting in the decrease in the grain size of the Ni-P-nano-tetragonal zirconia coatings. It caused the higher micro-hardness of the $\mathrm{Ni}-\mathrm{P}$-nano-tetragonal zirconia coatings [71]. Therefore, the dispersion hardening effect and the retardation in plastic deformation of base $\mathrm{Ni}-\mathrm{P}$ matrix due to the nano-tetragonal zirconia incorporation increased the micro-hardness of the coatings [72, 73]. All the coatings except bare $\mathrm{Ni}-\mathrm{P}$ exhibited comparatively better adhesion and wear resistance. The porosity of the electroless coatings was closely related to corrosion resistance [74]. It was examined by ferroxyl reagent test. Comparatively lesser of Prussian blue coloration was observed on the surface of the nano-tetragonal zirconia coatings, revealing its low porosity. The Ni-Pnano-tetragonal zirconia coating with an optimum amount exhibited good physicochemical characteristics than the other electroless coatings.

\subsubsection{Evaluation of Crystalline Characteristics of Ni-P- Nano-tetragonal Zirconia Coating}

The diffraction pattern of $\mathrm{Ni}-\mathrm{P}$-nano-tetragonal zirconia coating is compared with that of bare $\mathrm{Ni}-\mathrm{P}$ coating in Fig. 4A. In the XRD spectrum of the bare $\mathrm{Ni}-\mathrm{P}$ coating, a broad peak at 2 theta values $45^{\circ}$ and $65^{\circ}$ (JCPDS No.
00-045-1022) was observed which indicated the amorphous nature of the electroless Ni-P coating [70]. But the Ni-P-nano-tetragonal zirconia coating exhibited a partial crystalline nature which was evidenced from the narrower peak width obtained for $\mathrm{Ni}-\mathrm{P}$-nano-tetragonal zirconia coating than that of the bare $\mathrm{Ni}-\mathrm{P}$ coating. The microcrystalline XRD peaks of nickel atoms (JCPDS No. 00-045-1022) were observed at 2 theta $=45^{\circ}$ for both the coatings [75]. The peak corresponds to the reflection planes of a face-centered cubic phase (fcc) of nickel [70].

\subsubsection{Morphological and Topographical Characteristics of Ni-P-Nano-tetragonal Zirconia Coating}

SEM images of bare $\mathrm{Ni}-\mathrm{P}$ and $\mathrm{Ni}-\mathrm{P}-$ nano-tetragonal zirconia coatings are shown in Fig. 4D, E. Bare Ni-P and Ni$\mathrm{P}$-nano-tetragonal zirconia coatings were devoid of any pores/cracks on the surface. A uniform and homogeneous morphology was obtained for both the bare $\mathrm{Ni}-\mathrm{P}$ and the $\mathrm{Ni}-\mathrm{P}-$ nano-tetragonal zirconia coatings. The bare $\mathrm{Ni}-\mathrm{P}$ coating exhibited a uniform and regularly packed nodular amorphous structure [76], whereas a dense and smaller nodules were observed for the $\mathrm{Ni}-\mathrm{P}$-nano-tetragonal zirconia coating. It could be attributed to the agglomeration [77] of nano-tetragonal zirconia particles and the formation of larger number of nucleation sites on $\mathrm{Ni}-\mathrm{P}$ matrix due to the incorporation of nano-tetragonal zirconia. The developed Ni-P-nano-tetragonal zirconia coating was found to be dense and compact due to the uniform distribution of nucleation sites on the surface [76].

Topographical characteristics of the coatings were analyzed by AFM in contact mode. Figure S5 shows the 3D and $2 \mathrm{D}$ images of bare $\mathrm{Ni}-\mathrm{P}$ and $\mathrm{Ni}-\mathrm{P}-$ nano-tetragonal zirconia coatings. Both the coatings were found to be uniform without any cracks or defects. Nano-aggregates of the tetragonal zirconia were obvious in the AFM images of the $\mathrm{Ni}-\mathrm{P}-$ nano-tetragonal zirconia coating. AFM can also be used to measure the convexity of the coatings generated

Table 1 Comparison of physicochemical characteristics of varying amount of nano-tetragonal zirconia-incorporated Ni-P coatings

\begin{tabular}{|c|c|c|c|c|c|c|}
\hline $\begin{array}{l}\text { Sl. } \\
\text { no. }\end{array}$ & $\begin{array}{l}\text { Concentration of nano-tetragonal zirconia in the bath (g/ } \\
\mathrm{L})\end{array}$ & $\begin{array}{l}\text { Hardness } \\
\text { (VHN) }\end{array}$ & $\begin{array}{l}\text { Thickness } \\
(\mu \mathrm{m})\end{array}$ & Adhesion & Porosity & $\begin{array}{l}\text { Wear } \\
\text { resistance }\end{array}$ \\
\hline 1 & 0 & $458-463$ & $10-11$ & Good & porous & Fair \\
\hline 2 & 1 & $526-530$ & $11-12$ & Good & $\begin{array}{l}\text { Less } \\
\text { porous }\end{array}$ & Good \\
\hline 3 & 2 & $538-541$ & $12-14$ & Better & $\begin{array}{l}\text { Less } \\
\text { porous }\end{array}$ & Better \\
\hline 4 & 5 & $544-547$ & $14-15$ & Better & $\begin{array}{l}\text { Less } \\
\text { porous }\end{array}$ & Better \\
\hline 5 & 10 & $532-536$ & $12-13$ & Better & $\begin{array}{l}\text { Less } \\
\text { porous }\end{array}$ & Better \\
\hline
\end{tabular}



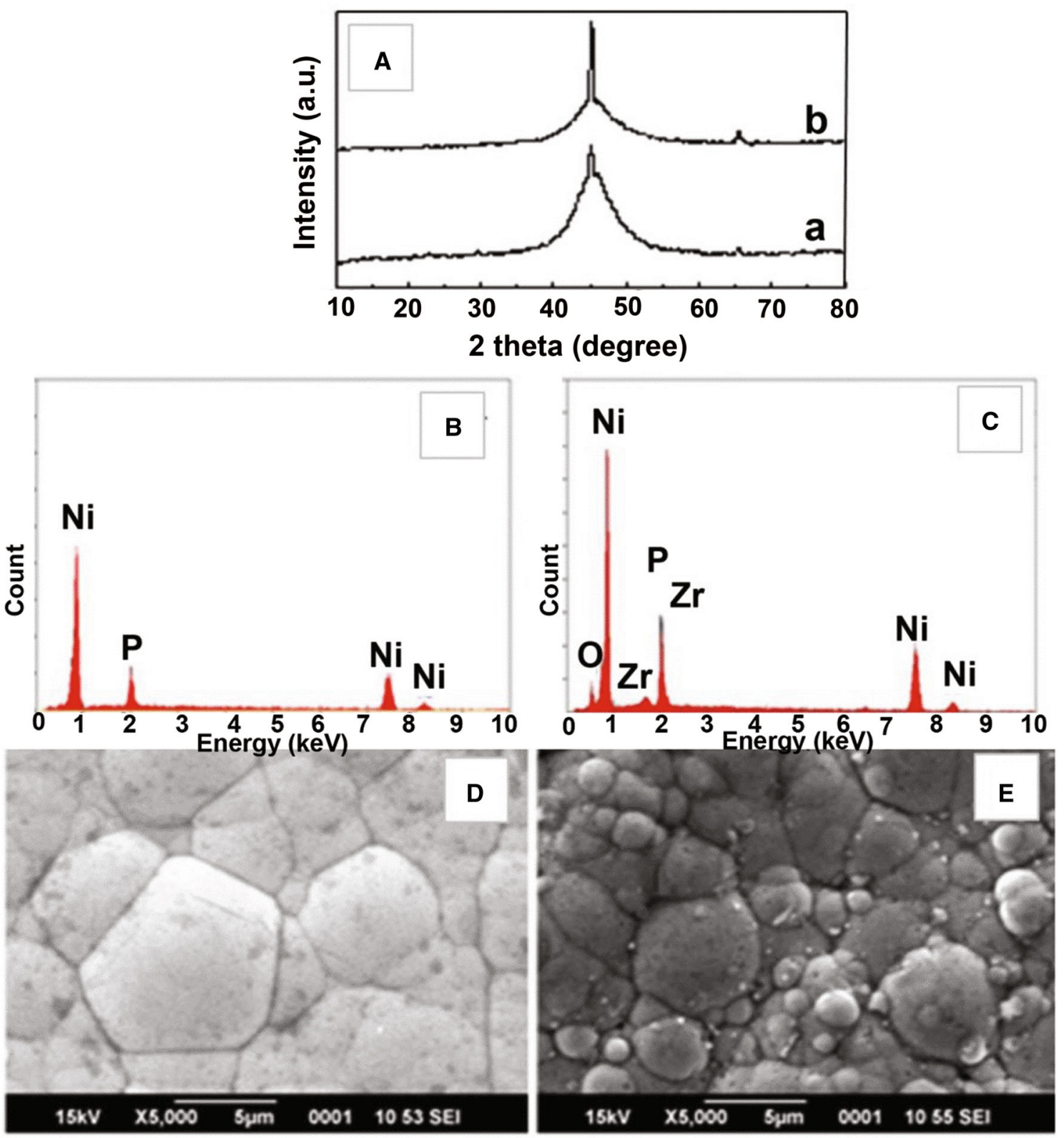

Fig. 4 A XRD patterns of (a) bare Ni-P and (b) Ni-P-nano-tetragonal zirconia coatings, B EDS spectra of bare Ni-P coating, C EDS spectra of Ni-P-nano-tetragonal zirconia coating, D SEM image of bare Ni-P coating, E SEM image of Ni-P-nano-tetragonal zirconia coating

due to the incorporation of nano-tetragonal zirconia particles [78]. The bare $\mathrm{Ni}-\mathrm{P}$ coating possessed large and hemispherical-shaped nodules, revealing a better smooth and compact surface morphology than the $\mathrm{Ni}-\mathrm{P}-$ nano-tetragonal zirconia coating. The Ni-P-nano-tetragonal zirconia coating exhibited a smaller grain size compared to that of bare Ni-P coating. Average surface roughnesses of the bare $\mathrm{Ni}-\mathrm{P}$ and the $\mathrm{Ni}-\mathrm{P}-$ nano-tetragonal zirconia coatings were obtained as $668 \mathrm{~nm}$ and $379 \mathrm{~nm}$, respectively. The high surface roughness generally results in a high surface-corrosive electrolyte contact and corrosion of the metals and metal-based alloys. It was reported that the rough metal and metal alloy surfaces can enhance the pitting susceptibility and general corrosion rate [79, 80]. In the present case, the incorporation of nano- $\mathrm{ZrO}_{2}$ into the $\mathrm{Ni}-\mathrm{P}$ matrix decreased the roughness of the coating and protected its surface contact with the electrolyte. A decrease in surface roughness by the incorporation of nanotetragonal zirconia into the bare $\mathrm{Ni}-\mathrm{P}$ matrix leads to an increase in the corrosion resistance characteristics. The grain growth was inhibited due to the uniform dispersion of nano-tetragonal zirconia particles in the $\mathrm{Ni}-\mathrm{P}$ matrix $[81,82]$. The Ni-P-nano-tetragonal zirconia coating appeared to be more crystalline with small nodular grains 
and clear nodular boundaries. However, agglomerations of the nano-tetragonal zirconia in the coating resulted in large nodular structures with some deep valley like appearance in some parts of the surface. Thus, the incorporation of nano-tetragonal zirconia into the Ni-P matrix had resulted in better quality coatings.

\subsubsection{Compositional Characteristics of the Ni-P-Nano- tetragonal Zirconia Coating}

Element composition, especially nano-tetragonal zirconia in the coatings, played a crucial role in the tribological and corrosion resistance behavior of the coatings [83-85]. The EDS spectra of bare Ni-P and Ni-P-nano-tetragonal zirconia coatings are shown in Fig. 4B, C. The EDS spectrum of the bare Ni-P contains the elemental peaks of nickel and phosphorous only, whereas the spectrum of Ni-P-nanotetragonal zirconia coating contains zirconium and oxygen, indicating the successful incorporation of nano-tetragonal zirconia into the Ni-P matrix. The absence of any other element peaks in the spectra evidenced the purity of the coatings. It also ensured the effectiveness of the selected electroless deposition bath and the operating conditions.

\subsection{Electrochemical Characterization of the $\mathrm{Ni}-$ P-Nano-tetragonal Zirconia Coating}

\subsubsection{Effect of Incorporation of Nano-tetragonal Zirconia for Long-Term Stability/Corrosion Protection of the Ni-P Coating}

The stability and corrosion resistance of the electroless $\mathrm{Ni}-$ $\mathrm{P}$ coatings were evaluated from the variations in the opencircuit potential (OCP) values of the coatings in 3.5\% (w/v) $\mathrm{NaCl}$ corrosive medium (Fig. S6). Both the bare Ni-P and $\mathrm{Ni}-\mathrm{P}-$ nano-tetragonal zirconia coatings exhibited an anodic shift in potential, which indicated the dissolution/disintegration of the surface of the coatings due to surface electrochemical reactions. However, the shift in potential was higher at the beginning of dissolution, but later the extent of potential shift decreased as the surface tended to attain a stable equilibrium condition. The bare Ni-P coating experienced a shift in potential within the range from -0.415 to $-0.616 \mathrm{~V}$, whereas in the case of $\mathrm{Ni}-\mathrm{P}-$ nanotetragonal zirconia coatings corresponding to the addition of $5 \mathrm{~g} / \mathrm{L}$ nano-tetragonal zirconia, the anodic shift in potential was lower within the range from -0.310 to $-0.466 \mathrm{~V}$. The other Ni-P-nano-tetragonal zirconia coatings experienced an anodic shift within a range of potential from -0.340 to $-0.520 \mathrm{~V}$. It revealed the better corrosion resistance achieved by the incorporation of nanotetragonal zirconia into the electroless $\mathrm{Ni}-\mathrm{P}$ coatings compared to bare Ni-P.

\subsubsection{Effect of Incorporation of Nano-tetragonal Zirconia on Anodic Polarization of Electroless Ni-P Coatings}

The polarization curves of the electroless $\mathrm{Ni}-\mathrm{P}$-nano-tetragonal zirconia coatings incorporated with varying amount of nano-tetragonal zirconia in $32 \%(\mathrm{w} / \mathrm{v}) \mathrm{NaCl}$ are shown in Fig. 5A. The coatings exhibited a similar trend in potential during the anodic polarization study. It might have caused by the similar type of fundamental reaction taking place on both the bare $\mathrm{Ni}-\mathrm{P}$ and electroless Ni-Pnano-tetragonal zirconia coatings. However, the shift in electrode potential with respect to the applied anodic current density was higher in the case of Ni-P-nano-tetragonal zirconia coatings compared to bare $\mathrm{Ni}-\mathrm{P}$ coating. It could be attributed to the improved corrosion resistance of the coatings. Among all the coatings, Ni-P-nano-tetragonal zirconia coating corresponding to the addition of $5 \mathrm{~g} / \mathrm{L}$ nano-tetragonal zirconia exhibited a higher and sudden shift in potential during anodic polarization due to the more passive and hence corrosion-resistant nature of the optimum Ni-P-nano-tetragonal zirconia coating.

\subsubsection{Tafel Polarization of the Ni-P-Nano-tetragonal Zirconia Coating}

The Tafel polarization curves of the bare $\mathrm{Ni}-\mathrm{P}$ and the $\mathrm{Ni}-$ $\mathrm{P}$-nano-tetragonal zirconia coatings corresponding to the addition of $5 \mathrm{~g} / \mathrm{L}$ nano-tetragonal zirconia are shown in Fig. 5B. The electrochemical parameters of the coatings were calculated from the Tafel curves listed in Table 2. The positive shift in corrosion potential, $E_{\text {corr }}$ and the decrease in corrosion current density of the Ni-P-nanotetragonal zirconia coating evidenced the enhanced corrosion resistance of the Ni-P-nano-tetragonal zirconia coating. The analysis of corrosion parameters indicated a similar trend in polarization resistance also. The Ni-Pnano-tetragonal zirconia coating experienced a higher polarization resistance of $13.200 \mathrm{k} \Omega / \mathrm{cm}^{2}$ with a lower corrosion current density of $3.909 \mu \mathrm{A} / \mathrm{cm}^{2}$. The calculated corrosion rate of Ni-P coating was found to decrease to $4.545 \times 10^{-2} \mathrm{~mm} /$ year on incorporation of nano-tetragonal zirconia into the base Ni-P matrix. This confirmed the improved corrosion resistance of Ni-P-nano-tetragonal zirconia coating. These results confirmed that the incorporation of nano-tetragonal zirconia had positive effects on the corrosion resistance characteristics of the Ni-P coating.

\subsection{Retention of Surface Characteristics of the Ni-P-Nano-tetragonal Zirconia Coating After Anodic Polarization}

The surface morphologies of the bare Ni-P and Ni-Pnano-tetragonal zirconia coatings after anodic polarization 

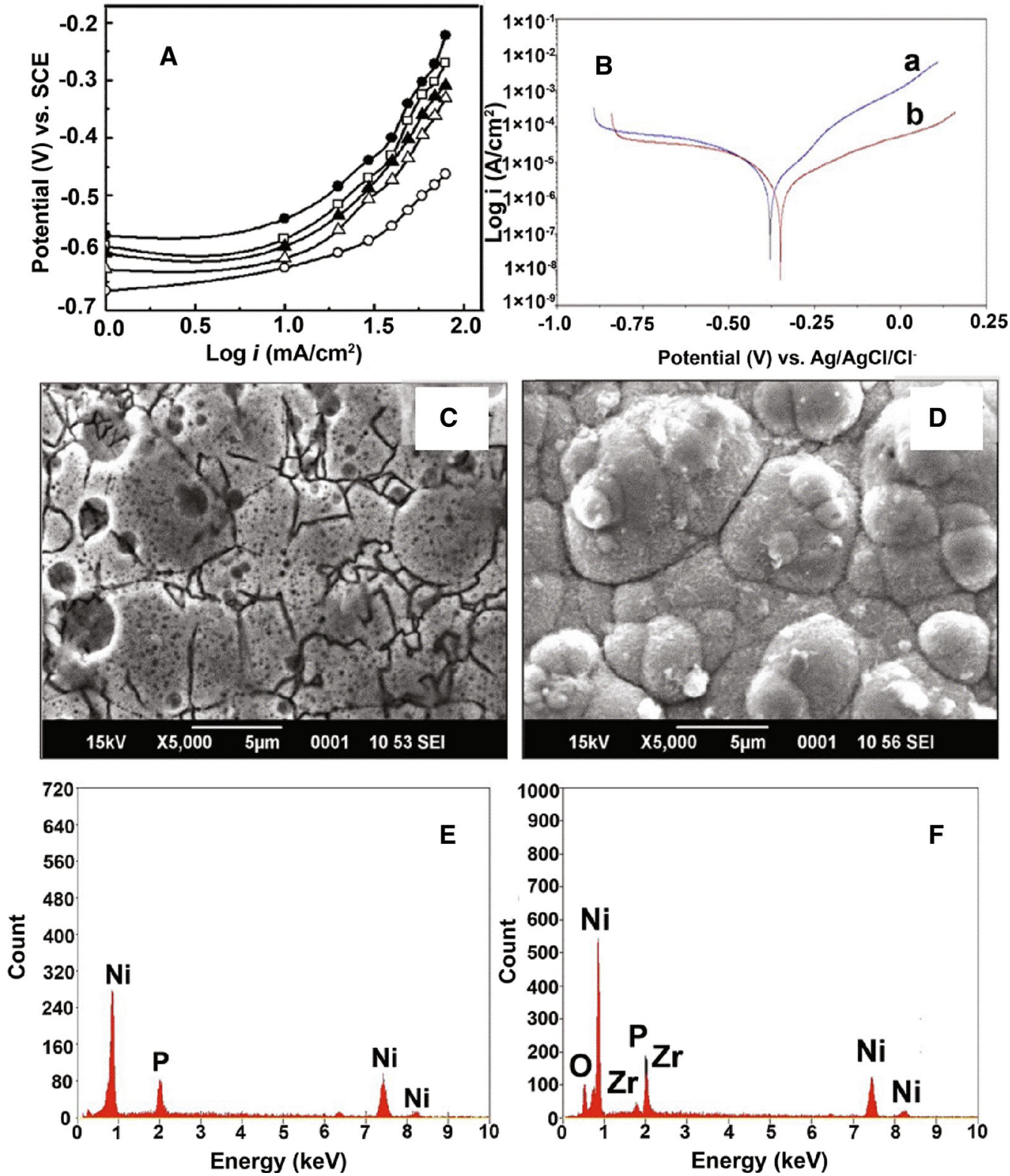

Fig. 5 A Anodic polarization curves of Ni-P-nano-tetragonal zirconia coatings in $3.5 \% \mathrm{NaCl}$ solution at temperature $30 \pm 2{ }^{\circ} \mathrm{C}$ [open circle$0 \mathrm{~g} / \mathrm{L}$, open triangle $-1 \mathrm{~g} / \mathrm{L}$, open square $-2 \mathrm{~g} / \mathrm{L}$, filled circle $-5 \mathrm{~g} / \mathrm{L}$ and filled triangle $-10 \mathrm{~g} / \mathrm{L}]$, B Tafel polarization curves of (a) bare Ni-P and (b) Ni-P-nano-tetragonal zirconia coatings, C SEM image of bare Ni-P after anodic polarization and D SEM image of Ni-P-nanotetragonal zirconia coating after anodic polarization, $\mathbf{E}$ EDS spectra of bare Ni-P coating after anodic polarization and $\mathbf{F}$ EDS spectra of Ni-Pnano-tetragonal zirconia coating after anodic polarization

Table 2 Comparison of corrosion characteristics of Ni-P coatings with and Ni-P-nano-tetragonal zirconia coating

\begin{tabular}{lllllll}
\hline Type of coating & $\beta_{\mathrm{a}}(\mathrm{V} / \mathrm{dec})$ & $\beta_{\mathrm{c}}(\mathrm{V} / \mathrm{dec})$ & $R_{\mathrm{p}}\left(\mathrm{k} \Omega / \mathrm{cm}^{2}\right)$ & $E_{\text {corr }}(\mathrm{V})$ & $i_{\text {corr }}\left(\mu \mathrm{A} / \mathrm{cm}^{2}\right)$ & Corrosion rate $(\mathrm{mm} / \mathrm{year})$ \\
\hline Bare Ni-P & 0.3078 & 0.1915 & 7.928 & -0.379 & 6.467 & $7.518 \times 10^{-2}$ \\
Ni-P-nano-tetragonal zirconia & 0.3828 & 0.1723 & 13.200 & -0.349 & 3.909 & $4.545 \times 10^{-2}$ \\
\hline
\end{tabular}

are shown in Fig. 5C, D. Many cracks and defects were observed on the bare Ni-P coating, revealing the removal of nodular surface of the bare Ni-P coating after anodic polarization. The Ni-P-nano-tetragonal zirconia coating exhibited no significant surface change, and also it retained its dense nodular structure even after anodic polarization. 
This attributed to the high corrosion protection ability of $\mathrm{Ni}-\mathrm{P}-$ nano-tetragonal zirconia coating.

The EDS spectra of bare Ni-P and Ni-P-nano-tetragonal zirconia coatings after anodic polarization are shown in Fig. 5E, F. After anodic polarization, the element composition of bare $\mathrm{Ni}-\mathrm{P}$ and $\mathrm{Ni}-\mathrm{P}-$ nano-tetragonal zirconia coatings was found to be almost similar as non-polarized coatings. The results revealed that the element composition was retained even though the bare Ni-P coating exhibited some cracks after polarization. Moreover, there was no peak of iron in the spectrum of bare $\mathrm{Ni}-\mathrm{P}$ or in the Ni-Pnano-tetragonal zirconia coatings after anodic polarization, revealing the high stability of the coatings. The peaks observed in the Ni-P-nano-tetragonal zirconia coating after polarization corresponded to zirconium, oxygen, nickel and phosphorous. The element composition of the coating before and after anodic polarization revealed that the coating had uniform element composition in its interior layers.

\subsection{Corrosion Behavior of Ni-P-Nano-tetragonal Zirconia Coatings Under Aggressive Conditions}

The corrosion protection of the coatings under aggressive condition was studied by salt spray analysis. The surface potential of the coatings during salt spray was monitored with respect to time and the graph as shown in Fig. S7. All the coatings exhibited a similar trend in variation of surface potential during salt spray analysis. The potential trends were as similar as trend in OCP variation. But the magnitude of potential shift was to a higher anodic region, revealing a higher corrosion attack on the surface of the coatings due to the high aggressiveness of the environment. Ni-P-nano-tetragonal zirconia coating corresponding to the addition of $5 \mathrm{~g} / \mathrm{L}$ nano-tetragonal zirconia exhibited a lower anodic shift in potential, revealing the higher corrosion protection than bare $\mathrm{Ni}-\mathrm{P}$ in aggressive conditions.

\subsection{Corrosion Behavior of Inner Layers of the Ni- P-Nano-tetragonal Zirconia Coating}

The anodic current densities of $20 \mathrm{~mA} / \mathrm{cm}^{2}$ and $50 \mathrm{~mA} /$ $\mathrm{cm}^{2}$ were applied on unit area of the coatings for $30 \mathrm{~min}$, and the corresponding anodic dissolution potential values were monitored. Different current densities resulted in a peeling off of the coating to different extents. The potential curves are shown in Fig. S8 (A) and (B). Bare Ni-P and the $\mathrm{Ni}-\mathrm{P}-$ nano-tetragonal zirconia coatings experienced a shift to anodic potential revealing the dissolution of the coatings in applied current densities. The bare Ni-P coating experienced a higher anodic shift in potential, whereas $\mathrm{Ni}-\mathrm{P}-$ nano-tetragonal zirconia coating corresponding to the addition of $5 \mathrm{~g} / \mathrm{L}$ nano-tetragonal zirconia exhibited lower shift in potential toward anodic region revealing the higher corrosion resistance.

OCP decay curves of the coatings after dissolution using $20 \mathrm{~mA} / \mathrm{cm}^{2}$ and $50 \mathrm{~mA} / \mathrm{cm}^{2}$ current densities are monitored and shown in Fig. S9 (A) and (B). A similar trend in potential shift was observed for all the coatings in both the cases. OCP variation of the coatings after dissolution was shifted to higher anodic region indicating that the surface reactions occurred at the electrode surface after dissolution. However, a stable and steady shift in potential toward anodic region indicated the uniform nature of the electroless coatings even after dissolution using $20 \mathrm{~mA} / \mathrm{cm}^{2}$ and $50 \mathrm{~mA} / \mathrm{cm}^{2}$ current densities. The dissolution of the coatings with higher current densities experienced a higher anodic shift in OCP. The bare Ni-P coating exhibited a higher anodic shift in potential in both the dissolution at different current densities, indicating that the corrosion reaction was more in the inner layer of the bare $\mathrm{Ni}-\mathrm{P}$ coating. The lesser anodic shift experienced by the Ni-Pnano-tetragonal zirconia coatings ensured the high corrosion resistance of the coating. The Ni-P-nano-tetragonal zirconia coating corresponding to the addition of $5 \mathrm{~g} / \mathrm{L}$ nano-tetragonal zirconia exhibited a lower and steady anodic shift in potential, revealing the uniform nature and higher corrosion resistance among the studied coatings. No sharp or rapid potential shift was observed in the coating revealing its uniformity in morphology and composition throughout the coating.

\subsection{Corrosion-Resistant Nature of Ni-P-Nano- tetragonal Zirconia Coating}

Nyquist and Bode plots of bare Ni-P and Ni-P-nano-tetragonal zirconia coatings in $3.5 \%(\mathrm{w} / \mathrm{v}) \mathrm{NaCl}$ are shown in Fig. 6A, C, respectively. The Nyquist plot consists of a semicircle section, indicating the charge transfer process, at a higher frequency region and a linear section, indicating the diffusion process/Warburg resistance, at the lower frequency region. The electron transfer resistance $R_{\mathrm{ct}} l$ conductivity is a direct measure of the semicircle diameter. The equivalent circuit model as depicted in Fig. 6B was used to fit the impedance plots, and thus, the obtained electrochemical parameters are enlisted in Table 3. The equivalent circuit consists of the circuit elements: $R_{1}$ solution resistance $\left(R_{\mathrm{S}}\right), R_{2}$-coating resistance, $R_{3}$ charge transfer resistance $\left(R_{\mathrm{ct}}\right), C_{1}$-coating capacitance and $C_{2}$-double-layer capacitance $\left(C_{\mathrm{dl}}\right)$. The bare Ni-P and $\mathrm{Ni}-\mathrm{P}-$ nano-tetragonal zirconia coatings exhibited a single semicircle in the high-frequency region, revealing that the same fundamental charge transfer process occurred on both the coatings but over a different effective area [71]. The 

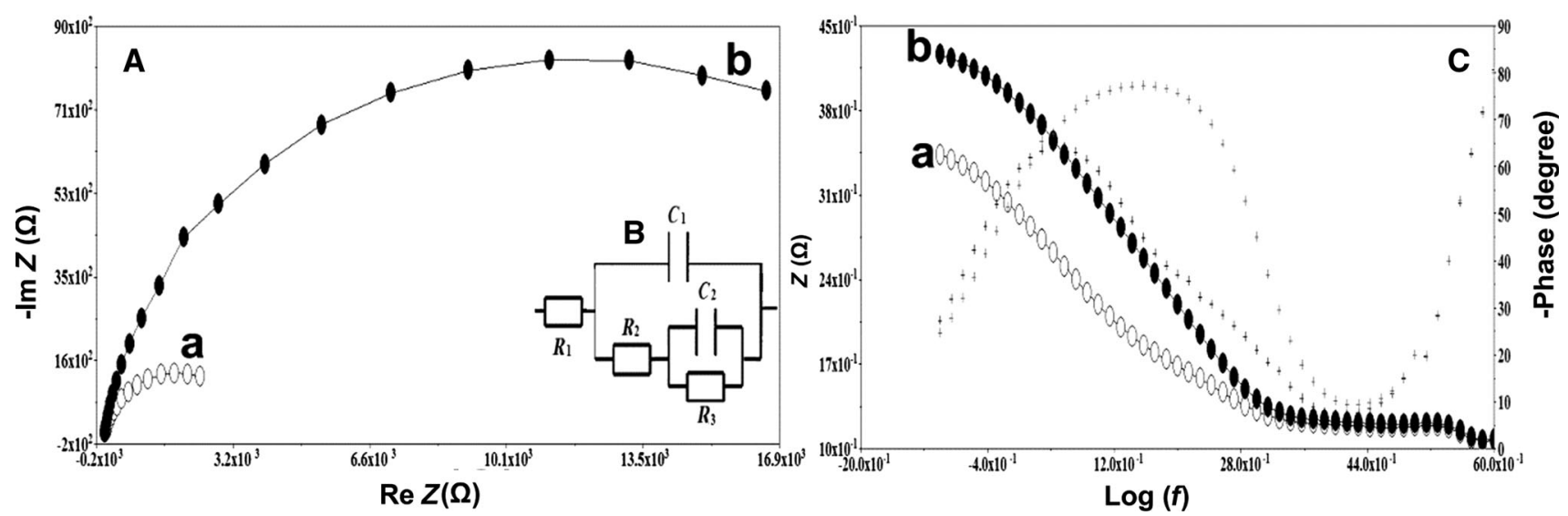

Fig. 6 A Nyquist plots of (a) bare Ni-P coating, (b) Ni-P-nano-tetragonal zirconia coating, B the circuit used to fit EIS data and $\mathbf{C}$ Bode plots (a) bare Ni-P coating and (b) Ni-P-nano-tetragonal zirconia coating

Table 3 Electrochemical parameters of electroless Ni-P coatings with and without nano-tetragonal zirconia incorporation in $3.5 \% \mathrm{NaCl}$ obtained from EIS analysis

\begin{tabular}{lll}
\hline Type of coating & $\mathrm{CPE}_{\mathrm{dl}}\left(\mu \mathrm{F} / \mathrm{cm}^{2}\right)$ & $R_{\mathrm{ct}}\left(\mathrm{k} \Omega / \mathrm{cm}^{2}\right)$ \\
\hline Pure Ni-P & 86.2 & 2.461 \\
Ni-P-nano-tetragonal zirconia & 11.56 & 16.75 \\
\hline
\end{tabular}

Nyquist plots with a single semicircle revealed chargecontrolled reaction occurring at the electrode surface of bare Ni-P and Ni-P-nano-tetragonal zirconia coatings.

Bode plots of bare $\mathrm{Ni}-\mathrm{P}$ and $\mathrm{Ni}-\mathrm{P}-$-nano-tetragonal zirconia coatings are shown in Fig. 6C. The single inflection point observed in the plot of $\log f$ vs. $\log |Z|$ for bare Ni-P and $\mathrm{Ni}-\mathrm{P}-$ nano-tetragonal zirconia coatings revealed that the charge transfer process involves only a single time constant. A single-phase angle maximum in the plot of log $f$ vs. phase angle confirmed the presence of a single time constant in the charge transfer process for both the bare $\mathrm{Ni}-$ $\mathrm{P}$ and Ni-P-nano-tetragonal zirconia coatings. Phase angle ' $\theta$ ' usually varies between a perfect capacitor $(n=1$ and $\left.\theta=90^{\circ}\right)$ and a perfect resistor $\left(n=0\right.$ and $\left.\theta=0^{\circ}\right)$. Phase angle maximum around $90^{\circ}$ of Ni-P-nano-tetragonal zirconia coatings indicated the pure capacitive behavior of the process. But bare Ni-P coating exhibited a phase angle maximum around $75^{\circ}$ indicating the non-homogeneous nature of the coating surface due to direct exposure to an aggressive medium [73]. Generally, the value of charge transfer resistance is inversely proportional to corrosion rate [86]. In the present case, the higher value of charge transfer resistance $\left(R_{\mathrm{ct}}=16.75 \mathrm{k} \Omega / \mathrm{cm}^{2}\right)$ and large radius of the semicircle obtained for Ni-P-nano-tetragonal zirconia coating confirmed high corrosion resistance of the coating. The double-layer capacitance also can be considered as a function of corrosion on the coating [87]. A low $C_{\mathrm{dl}}$ value exhibited by Ni-P-nano-tetragonal zirconia coating revealed the enhanced corrosion resistance due to improvement in surface homogeneity and low porosity of the coating.

\subsection{Corrosion Morphology of Electroless Ni-P- Nano-tetragonal Zirconia Coating After Immersion Test}

The SEM images of the bare Ni-P and Ni-P-nano-tetragonal zirconia coatings, after the immersion test, at different magnifications, are shown in Fig. S10. After the immersion test in $3.5 \%(\mathrm{w} / \mathrm{v}) \mathrm{NaCl}$ solution, a browncolored corrosion product was observed on bare $\mathrm{Ni}-\mathrm{P}$ revealing formation of localized corrosion sites at some regions, whereas in nano-tetragonal zirconia-Ni-P coatings, no such localized corrosion product was observed. The nodular morphology of bare Ni-P coating was damaged after the immersion test, whereas the nano-tetragonal zirconia-Ni-P coating almost retained its nodular grain structure even after exposure to a highly aggressive medium during the immersion test.

\section{Conclusion}

Nano-tetragonal zirconia of size 10-20 nm was prepared by a wet thermal decomposition method using zirconium oxychloride as the precursor. The zirconia retained its phase, bonding and electronic characteristics at high annealing temperature and in a reducing $\mathrm{Ni}-\mathrm{P}$ coating condition. The Ni-P-nano-tetragonal zirconia coating was developed for corrosion-resistive coating on mild steel surface. Physicochemical, topographical and morphological characteristics of the bare Ni-P coating on mild steel were enhanced by effective incorporation of nano- 
tetragonal zirconia. The long-term stability and corrosion protection characteristics of the Ni-P-nano-tetragonal zirconia coating with tuned nano-tetragonal zirconia content were significantly high under aggressive conditions. The $\mathrm{Ni}-\mathrm{P}-$ nano-tetragonal zirconia coating had retained uniform surface characteristics as evidenced from the results of anodic dissolution experiments. The coating had higher corrosion resistance as evidenced from the higher polarization resistance of $13.2 \mathrm{k} \Omega / \mathrm{cm}^{2}$, lower corrosion current density of $3.909 \mu \mathrm{A} / \mathrm{cm}^{2}$ and higher charge transfer resistance $\left(16.75 \mathrm{k} \Omega / \mathrm{cm}^{2}\right)$. The corrosion rate of Ni-P-nanotetragonal zirconia coating was as low as $4.545 \times 10^{-2}$ $\mathrm{mm}$ /year. The developed Ni-P-nano-tetragonal zirconia coating exhibited excellent physicochemical characteristics with the enhanced corrosion resistant characteristics.

Acknowledgement The authors are thankful to The Head, Faculties and researchers in Department of Chemistry, University of Kerala, Trivandrum, for their kind encouragement.

\section{References}

[1] D.R. Gabe, L. Chen, Trans. Inst. Met. Finish. 87, 8 (2009)

[2] A.S. Hamdy, M.A. Shoeib, H. Hady, Mater. Lett. 80, 191 (2012)

[3] G. Jiaqiang, W. Yating, L. Lei, H. Wenbin, Mater. Lett. 59, 391 (2005)

[4] H.L. Wang, L.Y. Liu, Y. Dou, W.Z. Zhang, W.F. Jiang, Appl. Surf. Sci. 286, 319 (2013)

[5] A. Ghasemi, T. Shahrabi, A. Ghasemi, T. Shahrabi, A.A. Oskuie, H. Hasannejad, S. Sanjabi, J. Alloy. Compd. 504, 237 (2010)

[6] M. Srivastava, V.K.W. Grips, A. Jain, K.S. Rajam, Surf. Coat. Technol. 202, 310 (2007)

[7] S.L. Wang, Surf. Coat. Technol. 186, 372 (2004)

[8] J. Novakovic, P. Vassiliou, K.I. Samara, T. Argyropoulos, Surf. Coat. Technol. 201, 895 (2006)

[9] E. Valova, J. Georgieva, S. Armyanov, I. Avramova, J. Dille, O. Kubova, D. Ogletree, Surf. Coat. Technol. 204, 2775 (2010)

[10] S. Sadreddini, A. Afshar, Appl. Surf. Sci. 303, 125 (2014)

[11] Y.Z. Zhang, Y.Y. Wu, K.N. Sun, M. Yao, J. Mater. Sci. Lett. 17, 119 (1998)

[12] C.F. Malfatti, H.M. Veit, C.B. Santos, M. Metzner, H. Hololeczek, J.P. Bonino, Tribol. Lett. 36, 165 (2009)

[13] G.S. Song, S. Sun, Z.C. Wang, C.Z. Luo, C.X. Pan, Acta Metall. Sin. (Engl. Lett.) 30, 1008 (2017)

[14] J.A. Wang, M.A. Valenzuela, J. Salmones, A. Vazquez, A.G. Ruiz, X. Bokhimi, Catal. Today 68, 21 (2001)

[15] L.E. Davies, N.A. Bonini, S. Locatelli, E.E. Gonzo, Latin Am. Appl. Res. 35, 23 (2005)

[16] Q. Wang, C. Li, M. Guo, C. Hu, Y. Xie, J. Mater. Chem. A 2, $1346(2014)$

[17] J.W. Dai, X.B. Zhang, Y. Fei, Z. Wang, H.M. Sui, Acta Metall. Sin. (Engl. Lett.) 31, 865 (2018)

[18] A. Hajalilou, M. Hashim, H.M. Kamari, K. Javadi, S. Kanagesan, M. Parastegari, Acta Metall. Sin. (Engl. Lett.) 27, 1144 (2014)

[19] S.E.H. Martínez, J.J.C. Rivera, R.M. Sánchez, C.G.G. Reyes, J.A.M. Bolanos, J.M. Cabrera, J.L.H. Rivera, Acta Metall. Sin. (Engl. Lett.) 29, 895 (2016)
[20] G. Dercz, K. Prusik, L. Pajak, J. Archiev. Mater. Manuf. Eng. 31, 414 (2008)

[21] B.K. Kim, J.W. Hahn, K.R. Han, J. Mater. Sci. Lett. 16, 669 (1997)

[22] F. Lu, J. Wang, M. Lang, M. Toulemonde, F. Namavar, C. Trautmann, J. Zhang, R.C. Ewing, J. Lian, Phys. Chem. Chem. Phys. 14, 12295 (2012)

[23] R. Suyama, T. Ashida, S. Kume, J. Am. Ceram. Soc. 68, 314 (1985)

[24] R.C. Garvie, R.H. Hannink, R.T. Pascoe, Nature 258, 703 (1975)

[25] S. Jayakumar, P.V. Ananthapadmanabhan, T.K. Thiyagarajan, K. Perumal, S.C. Mishra, G. Suresh, L.T. Su, A.I.Y. Tok, Mater. Chem. Phys. 140, 176 (2013)

[26] M. Signoretto, A. Breda, F. Somma, Microporous Mesoporous Mater. 91, 23 (2006)

[27] M.A. David, Microporous Mesoporous Mater. 28, 505 (1999)

[28] M. Li, Z. Feng, P. Ying, Q. Xin, C. Li, Phys. Chem. Chem. Phys. 5, $5326(2003)$

[29] F.Y. Lu, J.M. Zhang, M.B. Huang, F. Namavar, R.C. Ewing, J. Lian, J. Phys. Chem. C 115, 7193 (2011)

[30] S. Cavalu, F. Banica, V. Simon, I. Akin, G. Goller, Int. J. Appl. Ceram. Technol. 11, 402 (2014)

[31] A. Saxena, G. Aeppli, MRS Bull. 34, 804 (2009)

[32] S. Shukla, S. Seal, R. Vij, S. Bandyopadhyay, Z. Rahman, Nano Lett. 2, 989 (2002)

[33] T. Charaska, A.H. King, C.C. Berndt, Mater. Sci. Eng. A 286, 169 (2000)

[34] A. Ghoufi, G. Maurin, G.J. Ferey, Phys. Chem. Lett. 1, 2810 (2010)

[35] C.T. Lee, M.S. Zhang, Z. Yin, J. Mater. Sci. 43, 2675 (2008)

[36] Q.F. Zhou, H.L.W. Chang, Q.Q. Zhang, C.L. Choy, J. Appl. Phys. 89, 8121 (2001)

[37] G.D. Wilk, R.M. Wallace, J. Anthony, J. Appl. Phys. 89, 5243 (2001)

[38] M.W. Pitcher, S.V. Ushakov, A. Navrotsky, B.F. Woodfield, G. Li, J.B. Goates, B.M. Tissue, J. Am. Ceram. Soc. 88, 160 (2005)

[39] Q. Wang, C. Li, M. Guo, C. Hu, Y. Xie, J. Mater. Chem. A 2, 1346 (2014)

[40] Y. Yang, W. Chen, C. Zhou, H. Xu, W. Gao, Appl. Nanosci. 1, 19 (2011)

[41] B. Szczygieł, A. Turkiewicz, J. Serafinczuk, Surf. Coat. Technol. 202, 1904 (2008)

[42] K. Zielinska, A. Stankiewicz, I. Szczygieł, J. Colloid Interf. Sci. 377, 362 (2012)

[43] Y.W. Song, D.Y. Shan, R.S. Chen, E.H. Han, Surf. Eng. 23, 334 (2007)

[44] S.M.A. Shibli, G.J. Harikrishnan, V.R. Anupama, K.S. Chinchu, B.N. Meena, Surf. Coat. Technol. 262, 48 (2015)

[45] S.S.A. Rehim, A.M.I. Magdy, K.F. Khaled, J. Appl. Electrochem. 29, 593 (1999)

[46] E.L. Hibner, Nickel (and alloys), in Corrosion Tests and Standards, Application and Interpretation, ed. by R. Baboain (ASTM International, West Conshohocken, 2005), pp. 580-584

[47] L.L. Wong, S.I. Martin and B. Rebak, Methods to calculate corrosion rates for alloy 22 from polarization resistance experiments, in Proceedings of PVP2006 2006 ASME Pressure Vessels and Piping Division Conference, Vancouver, PVP2006ICPVT11-93421, 23-27 July 2006

[48] G.D. Vishwanath, G.A. Yusuf, Microporous Mesoporous Mater. 1, 88 (2012)

[49] Y. Yin, A.P. Alivisatos, Nature 437, 664 (2005)

[50] G. Duan, C. Zhang, A. Li, X. Yang, L. Lu, X. Wang, Nanoscale Res. Lett. 3, 118 (2008)

[51] H.P. Klug, L.E. Alexander, X-ray Diffraction Procedures, 2nd edn. (Wiley, New York, 1975), p. 553 
[52] R. Srinivasan, R.J.D. Angelis, G. Ice, B.H. Davis, J. Mater. Res. 6, 1287 (1991)

[53] R. Stevens, Zirconia and Zirconia Ceramics, 2nd edn. (Magnesium Elektron Publication, Magnesium Elektron Ltd, Twickenham, 1986), p. 113

[54] R.A. Miller, R.G. Garlick, J.L. Smialek, J. Am. Ceram. Soc. Bull. 62, 1355 (1983)

[55] A. Benedetti, G. Fagherazzi, F. Pinna, J. Am. Ceram. Soc. 72, 467 (1989)

[56] T.K. Gupta, J.H. Bechtold, R.C. Kuznicki, L.H. Cadoff, B.R. Rossing, J. Mater. Sci. 12, 2421 (1977)

[57] K.K. Srivastava, R.N. Patil, C.B. Choudhary, K.V. Gokhale, E.C.R. Subba, Trans. Br Ceram. Soc. 73, 85 (1974)

[58] Z. Sherafat, I. Antunes, C. Almeida, J.R. Frade, M.H. Paydar, G.C. Mather, D.P. Fagg, Dalton Trans. 43, 9324 (2014)

[59] C. Prakash, S.D. Dhananjay, B.U. Shubhangi, V.B. Ankush, J. Mater. Chem. A 2, 19060 (2014)

[60] A.D. Brande, R. Perezzan, E.U. Garrote, L.C. Otero-Diaz, Inorg. Chem. 50, 4640 (2011)

[61] R.C. Garvie, J. Phys. Chem. 69, 1238 (1965)

[62] M. Li, Z. Feng, G. Xiong, P. Ying, Q. Xin, C. Li, J. Phys. Chem. B 105, 8107 (2001)

[63] J.A. Navio, M.C. Hidalgo, G. Colon, S.G. Botta, M.I. Litter, Langmuir 17, 202 (2001)

[64] N.C.S. Selvam, A. Manikandan, L.J. Kennedy, J.J. Vijaya, J. Colloid Interf. Sci. 389, 91 (2013)

[65] M. Xiao, Y. Li, Y. Lu, Z. Ye, J. Mater. Chem. A 3, 2701 (2015)

[66] Y. Hao, J. Li, X. Yang, X. Wang, L. Lu, Mater. Sci. Eng. A 367, 243 (2004)

[67] F. Chen, Q. Hong, G.Q. Xu, T.S.A. Hor, S. Shen, J. Am. Ceram. Soc. 88, 2649 (2005)

[68] J. Ma, J. Zhang, S. Wang, T. Wang, J. Lian, X. Duan, W. Zheng, J. Phys. Chem. C 115, 18157 (2011)

[69] I. Ferino, M.F. Casula, A. Corrias, M.G. Cutrufello, R. Monaci, G. Paschina, Phys. Chem. Chem. Phys. 2, 1847 (2000)
[70] S.A. Gawad, A.M. Baraka, M.S. Morsi, M.A. Eltoum, Int. J. Electrochem. Sci. 8, 1722 (2013)

[71] R. Arghavanian, N.P. Ahmadi, S. Yazdani, B. Bostani, Surf. Eng. 28, 503 (2012)

[72] C. Ma, F. Wu, Y. Ning, F. Xia, Y. Liu, Ceram. Int. 40, 9279 (2014)

[73] Z.A. Hamid, S.A.E. Badry, A.A. Aal, Surf. Coat. Technol. 201, 5948 (2007)

[74] J.N. Balaraju, V.E. Selvi, V.W. Grips, K.S. Rajam, Electrochim. Acta 52, 1064 (2006)

[75] S.W. Tang, C.J. Wang, Y.L. Sun, J. Hu, Surf. Coat. Technol. 205, 43 (2010)

[76] T.S. Narayanan, I. Baskaran, K. Krishnaveni, S. Parthiban, Surf. Coat. Technol. 200, 3438 (2006)

[77] W. Chen, W. Gao, Y. He, Surf. Coat. Technol. 204, 2493 (2010)

[78] M.F. Montemor, W. Trabelsi, S.V. Lamaka, K.A. Yasakau, M.L. Zheludkevich, A.C. Bastos, M.G.S. Ferreira, Electrochim. Acta 53, 5913 (2008)

[79] R. Walter, M.B. Kannan, Mater. Des. 32, 2350 (2011)

[80] A. Shahryari, W. Kamal, S. Omanovic, Mater. Lett. 62, 3906 (2008)

[81] M.H. Wu, Z. Li, F.F. Xia, L.Y. Chen, Mater. Mech. Eng. 29, 58 (2005)

[82] F.F. Xia, C. Liu, F. Wang, M.H. Wu, J.D. Wang, H.L. Fu, J.X. Wang, J. Alloys Compd. 490, 431 (2010)

[83] S. Ranganatha, T.V. Venkatesha, K. Vathsala, Appl. Surf. Sci. 256, 7377 (2010)

[84] J.N. Balaraju, V.E. Selvi, K.S. Rajam, Mater. Chem. Phys. 120, 546 (2010)

[85] J.N. Balaraju, C. Anandan, K.S. Rajam, Surf. Coat. Technol. 200, 3675 (2006)

[86] L. Fan, F. Tang, S.T. Reis, G. Chen, M.L. Koenigstein, Acta Metall. Sin. (Engl. Lett.) 30, 390 (2017)

[87] Y.Z. Mao, Y.H. Wei, H.T. Zhao, C.X. Lv, H.J. Cao, J. Li, Acta Metall. Sin. (Engl. Lett.) 31, 1171 (2018) 